\title{
Enumeration Processes under Attack: The Role of Working Memory in Subitizing and Serial Counting
}

\section{Sarit Ashkenazi*}

Learning Disabilities, School of Education, The Hebrew University of Jerusalem, Mount Scopus, Jerusalem

\begin{abstract}
Enumeration of visual stimuli is supported by two distinct psychological processes: serial counting and subitizing. Serial counting is an effortful, slow, and controlled process employed for the enumeration of large sets of objects. Subitizing is defined as a fast and accurate assessment of small quantities. Over the past two decades, there has been an on-going debate on whether subitizing and serial counting are based on shared or distinct cognitive mechanisms. Recent theories suggest that subitizing is supported by visual skills related to perception while serial counting requires working memory.

The current study examines the respective roles of phonological and spatial working memory in enumeration processes. The main task used was an enumeration task, in which participants named the quantities of randomly arranged dots in the subitizing (1-3 dots) and counting (7-9 dots) ranges. The performance in the enumeration naming task was compared to a dual-task setting in which participants performed the enumeration naming task while they retained a phonological load or a spatial load.

Load type had differential impact on enumeration processes. Importantly, it was found that phonological load, but not spatial load, decreased the effectiveness of serial counting. Subitizing ability was not affected by spatial or phonological load. In line with the majority of previous studies on enumeration, our results indicate that enumeration of small and large quantities are based on different cognitive processes. Moreover, the present finding demonstrate that, phonological working memory plays a significant role in serial counting but not in subitizing and that spatial load is not involved in enumeration.
\end{abstract}

Keywords: Enumeration; Working memory; Subitizing; Counting; dual-task; Phonological load; Spatial load

\section{Introduction}

Enumeration of visual stimuli is supported by two, or more, distinct psychological processes. 1) Serial counting, an effortful, slow, and controlled process that is engaged when counting relatively large sets of objects (more than 4). Reaction time (RT) slope for serial counting increases linearly, as the number of items increases RT increases as well [1-4]. 2) Subitizing is a fast and accurate assessment of small quantities [5], the subitizing range is between 1 to 3 (or 4) items.

As opposed to serial counting, RT barely increases (100 ms per item) as items increase within the subitizing range, and therefore, the slope remains relatively flat [1-4]. Serial counting is more complex compared to subitizing and involves many processes including: individualization and localization of objects, switching spatial attention from one object to the other, summing the number of objects and inhibition of already summed objects [4].

\section{Various enumeration processes are supported by distinct cognitive mechanisms}

Over the last two decades, different perspectives on the cognitive mechanisms underlying enumeration processes have emerged $[6,7]$. The single process hypothesis suggests that there is no qualitative difference between the enumeration of small and large objects, and that subitizing is identical to the process of fast counting [7]. However, according to the magnitude hypothesis, subitizing is supported by the analog magnitude system, similar to estimation and operates according to Weber's law [6].

Moreover, the canonical enumeration theory suggests that subitizing and serial counting are supported by distinct cognitive mechanisms. Several findings in research support the canonical enumeration theory. Studies on quantitative understanding in young infants have demonstrated the existence of two distinct systems: one for a small exact number of objects and another for numerical estimation (i.e., larger amount of objects) $[8,9]$.

In line with this hypothesis, a study comparing adult performance on an estimation task found that while accuracy and variability of estimations of quantities larger than 10 followed Weber's law, estimation of quantities in the subitizing range (between 1 to 4 ) did not, suggesting a dissociation of domain specific estimation processes from subitizing [10]. Importantly, later, the same authors [11] proposed that subitizing reflects visuo-spatial object individuation capacity, by demonstrating that individual differences in subitizing capacity related to individual differences in visuo-spatial object individuation capacity.

Moreover, Trick and Pylyshyn [4] suggested that small (subitizing range) and large (counting range) numbers are enumerated differently. They argued that subitizing employs a limited-capacity parallel mechanism for item individuation, associated with the multiple targets tracking task $[12,13]$. Hence, subitizing relies on preattentive information, whereas serial counting requires spatial attention. Since

*Corresponding author: Learning Disabilities, school of Education, The Hebrew University of Jerusalem, Mount Scopus, Jerusalem 91905, Tel: 97225882058; E-mail: sarit.ashkenazi@mail.huji.ac.il

Received January 12, 2016; Accepted February 12, 2016; Published February 20, 2016

Citation: Ashkenazi S (2016) Enumeration Processes under Attack: The Role of Working Memory in Subitizing and Serial Counting. J Foren Psy 1: 103. doi: 10.4172/2475-319X.1000103

Copyright: ( 2016 Ashkenazi S. This is an open-access article distributed under the terms of the Creative Commons Attribution License, which permits unrestricted use, distribution, and reproduction in any medium, provided the original author and source are credited. 
then, studies have found contradictory findings by highlighting the role of attention in subitizing. For example, studies have shown that during dual tasks, when spatial attention is diverted from the estimation task, subitizing suffers [14].

\section{Working Memory and Enumeration Processes}

Another line of thinking points to the different involvement of working memory capacities in subitizing and counting, hence, demonstrating the dissociation between enumerations processes. For example, participants with high working memory abilities had steeper counting slope than participants with low working memory abilities, while the subitizing slope was comparable between the two groups [15].

In that study, working memory was defined as a unified system [15]. However, a common conceptualization of working memory is based on Baddeley's model $[16,17]$ that proposed a partitioning of working memory into three components, namely the central executive, phonological loop, and visual-spatial sketchpad. The multicomponent view of working memory proposes a general cognitive model for the storage and manipulation of information that includes a central executive for high level monitoring and control, and a subsidiary phonological loop and visuo-spatial sketchpad for short term storage and maintenance of verbal, and visuo-spatial information, respectively [16-20]. Additional models suggested that visuo-spatial working memory can be broken down further into two unique systems one for visual and one for spatial information. In fact, dissociations can be found between visual (e.g. remember the shape of an object) and spatial (e.g. remember the location of an object) working memory tasks $[21,22]$.

\section{The dual-task paradigm as a common tool for investigating the role of working memory}

A frequently used approach for studying the role of the working memory sub systems is the dual-task paradigm in which a participant engages in two tasks simultaneously that engage working memory [23]. Classically, there is the main task of interest to the study and a secondary task whose purpose is to create a strain on working memory. The secondary task is usually started before the main task and finished after the main task.

If the two tasks involve the same cognitive resource than the performance in the main task would be affected by the secondary task and performance in the dual task condition would be inferior to the performance of the main task in the single task condition. It is very common to use working memory tasks as the secondary task in order to expose the involvement of different working memory components in cognitive tasks. For example, articulatory suppression, a secondary task that involves repeating words or syllable (e.g., saying "one one one") is believed to demand verbal working memory or the phonological loop but not the visuospatial working memory [24].

In Trick's [25] study, participants carried out an enumeration task, which included trials in the both the subitizing and the counting range, while simultaneously carrying out one of the following secondary tasks: articulation or finger tapping. In the subitizing range, the tapping task produced more interference than the articulation task. However, in the counting range both articulation and tapping tasks produced similar amount of interference. The authors concluded that different processes are involved in subitizing and serial counting, with phonological memory playing a larger role in serial counting.

Interestingly, Piazza et al. [11] examined the role of visual working memory in subitizing and estimation by performing a dual-task paradigm during subitizing and estimation. The results indicated that subitizing, but not estimation, was negatively impacted from a visual working memory load.

\section{The present study}

In light of the current theories which suggest that visuo-spatial working memory is strongly involved in subitizing, we evaluated enumeration in the subitizing and counting ranges under two different load conditions: spatial load and phonological load. The main task used was an enumeration task, in which participants named the quantity of randomly arranged dots in the subitizing (1-3 dots) or the counting (7-9 dots) range. The performance in the enumeration task was compared to a dual-task setting in which participants performed the enumeration task while they retained a phonological load (i.e., a non-words composed from three letters; e.g., Trbovich \& LeFevre, [26] or a spatial load (i.e., a computerized version of the Corsi Block task, e.g., Berch, Krikorian, \& Huha, [27]. The effect of load condition was examined by comparing the slope and performance in subitizing and counting ranges in the three conditions: the enumeration task alone, and two dual task conditions, phonological load and spatial load.

\section{Method}

\section{Participants}

Twenty-one students from the Hebrew University of Jerusalem, 18 female and 3 males, participated in the experiment. They were between the ages of 19 and 26 years old $(\mathrm{M}=24.36)$. All the participants were right-handed and had normal or corrected-to-normal visual acuity. They were paid with course credits or 30 NIS (approximately 8.6\$).

The study was approved by the local Ethics Committee of the School of education in the Hebrew university of Jerusalem. Written consent was obtained according to the Declaration of Helsinki. The participants provided written informed consent.

\section{Apparatus}

All the experimental tasks were controlled by a Genuine-Intel compatible PC $1.73 \mathrm{GHz}$ using E-prime experimental software, 2.1 versions (Schneider, Eschman, \& Zuccolotto, 2002). Instructions and stimuli were presented on a 17 " monitor screen. The computer monitor was located at approximately $50 \mathrm{~cm}$ in front of the subject. The keyboard and microphones were used.

\section{Materials and Procedure}

All participants performed the enumeration task in three conditions: single task (only enumeration), dual task- enumeration task with phonological memory load, and dual task- enumeration task with spatial memory. Before performing the dual tasks, participants' phonological and spatial spans were measured. The order of the tasks

\section{Single task}

Numbers from 1 to 3 (subitizing range) and 7 to 9 (counting range) were presented on the computer screen. On each trial, participants were presented with a group of dots at the center of a screen. They were asked to say aloud the number of presented dots as quickly as possible without making mistakes. The arrangement of dots was randomized. The dots were presented in white on a black background. Vocal RTs and errors were recorded. Vocal RTs were input via a microphone and were recorded electronically by a response box controlled by E-prime software. RT was measured from onset of the stimulus to onset of the vocal response. 
A typical trial started with a fixation asterisk for $300 \mathrm{~ms}$, followed by a blank screen for $500 \mathrm{~ms}$, a stimulus response, and a blank screen until the experimenter keyed in the participant's response. The inter-trial interval (from the experimenter's key-press to the onset of fixation of the next trial) was $1500 \mathrm{~ms}$. In order to make the single task condition as similar as possible to the dual task, each block was divided into six sub-blocks, with breaks between them. In each sub-block, there were 16 experimental trials. This resulted in 96 trials per block.

\section{Spatial span test}

A computerized version of the Corsi Block test was used to measure the participants' visuo-spatial working memory span. Participants had to reproduce the sequence in which squares were light up. There were nine blue squares $(2 \mathrm{~cm} \times 2 \mathrm{~cm})$ unevenly distributed over a $16 \mathrm{~cm} \times 16$ $\mathrm{cm}$ quadrant on the computer screen. The positions of the squares were fixed. Each trial began with the presentation of the nine squares in blue and next, a sequence of squares light up in yellow at a rate of one square per second. The squares remained on the screen until $500 \mathrm{~ms}$ after the sequence was completed. Then, a black screen was presented during 15 s. The nine blue squares were presented again and the participant had to reproduce the sequence in the order in which the squares light up by mouse click.

The starting level of the task was a sequence of two squares. If a participant successfully reproduced the sequence of two squares for two trials the difficulty was raised to a sequence of three squares ranging until nine squares. Each difficulty level included two trials. The difficulty progressively increased the sequence length if the subject gave the correct sequence in two out of two trials. The subject's span was recorded as the longest sequence he/she was able to successfully reproduce for two successive trials.

\section{Phonological span test}

To measure the phonological span triplets of pseudo-words were used. The triplets were constructed so that they did not spell a word in Hebrew. We constructed lists of two to six triplets. Each pseudo word combination was used only once during the experiment. In a trial, a pseudo word was presented sequentially for 2 seconds each on a computer, followed by the instructions: «please repeat the letter sequences». The data was collected by the experimenter on a sheet of paper.

The starting level was two triplets. There were two trials in each level. The difficulty level was raised by increasing the number of triplets if the subject was correct in at least two out of the two trails. The subject's span was the largest number of triplets in which two lists were correctly reproduced.

\section{Enumeration task with spatial memory load}

The structure of this dual task was similar to the single task with only enumeration but, at the beginning of each block, a sequence of squares to be recalled was presented. The number of squares in the sequence was fitted to the participants' spatial memory span. The events on each block were as follows: first, the nine squares were presented, then a sequence of squares light up, followed by the enumeration task for 16 trials. At the end of each block, a sequence was presented and the subjects had to indicate whether the sequence was identical to the one that was presented at the beginning of the block.

\section{Enumeration task with phonological memory load}

This task was similar to the single task condition, but included at the beginning of each block a set of non-word triplets to be recalled. The number of triplets was fitted to the participants' phonological span. The sequence of events on each block was equated to the spatial memory load condition.

At the end of each block, a sequence was presented and the subjects had to indicate whether the sequence was identical to the one that was presented at the beginning of the block.

\section{Results}

\section{Memory load tasks}

Subjects' visuo-spatial span ranged from 3 to $7(\mathrm{M}=5.09)$. Phonological spans were between 2 and $4(\mathrm{M}=3)$. For each participant, we computed the proportion of blocks in which correct responses were given to the memory task. In the phonological load, the averaged proportion of correct responses was 0.98 (S.D. $=0.04$ ); and in the spatial load the average was $0.87(\mathrm{SE}=0.10)$. The difference in accuracy rates between these two tasks was significant, $t(20)=-4.5$, $\mathrm{p}<0.001$. In addition, we correlated for each block and each subject the mean RT accuracy rates in the enumeration task and the mean RT accuracy in the load task. Accuracy was measured as the proportion of squares correctly responded in the correct order for spatial load and the proportion of triplets correctly answer for phonological load. The correlation was not significant in either the dual- spatial condition $(\mathrm{r}$ $=-0.06$ for accuracy and $\mathrm{r}=-0.01$ for RT) or the dual-phonological condition $(r=0.15$ for accuracy and $r=0.03$ for RT). Therefore, there was no trade-off between the tasks.

\section{Efficacy measurement}

We combined the results of the error rate and reaction time measurements. First, we calculated a standardized score of each one of the measurements, and then we calculated an average score of the two measurements. Lower scores indicated lower error rates and faster reaction times.

A three-way analysis of variance (ANOVA) was performed with load condition (no load, spatial load and phonological load) range (subitizing /counting) and quantity (small, medium, and large), as within participant factors.

The effects of range, $F(1,20)=428$, partial $\eta^{2}=0.96, p<.01$, and quantity, $\mathrm{F}(2,40)=23.31$, partial $\eta^{2}=0.54, \mathrm{p}<0.01$, were significant; participants performed better in the subitizing range (-0.31 S.D. $0.28)$ than the counting range (1.04 S.D. 0.53). Quantity and range interacted, $\mathrm{F}(2,40)=33.63$, partial $\eta^{2}=0.62, \mathrm{p}<0.01$. Quantity effected performance in the counting range, $\mathrm{F}(2,40)=34.87$, partial $\eta^{2}=0.64, \mathrm{p}$ $<0.01$, but not the subitizing range $(\mathrm{p}=0.14)$.

Importantly, the effect of load was significant $F(2,40)=13.04$, partial $\eta^{2}=0.40, p<0.01$. Participants were performed better in the no load condition (0.29 S.D. 0.39) compared with the phonological load condition (0.49 S.D. 0.37) F $(1,20)=30.82$, partial $\eta^{2}=.61, p<.01$. However, participants had comparable performances during spatial load (0.34 S.D. 0.46) and no load (0.29 S.D. 0.39) conditions $F(1,20)$ $=1.1$, partial $\eta^{2}=0.05, \mathrm{p}=0.31$.

Moreover, load and range interacted $\mathrm{F}(2,40)=13.04$, partial $\eta^{2}=$ $0.40, \mathrm{p}<.01$ and load and quantity interacted $\mathrm{F}(4,80)=4.8$, partial $\eta^{2}=$ $0.19, \mathrm{p}<0.01$. Accordingly, we analyzed each of the loads in every one of the conditions. In the subitizing range phonological load and spatial load did not affect performance $F(1,20)=0.75$, partial $\eta^{2}=0.04, p=$ 0.79 and $F(1,20)=0.02$, partial $\eta^{2}=0.00, p=0.91$ respectively. 
However, in the counting range, participants had better performance in the no load condition (0.88 S.D. 0.48) compared with the phonological load condition (1.28 S.D. 0.48$) ; \mathrm{F}(1,20)=35.5$, partial $\eta^{2}=0.64, p<0.01$ and the slope for the phonological load (0.57 S.D. 0.27 ) was higher than the slope of the no load condition (0.22 S.D. 0.20) $\mathrm{T}(21)=-4.5, \mathrm{p}<0.01$. However, spatial load (0.99 S.D. 0.62) and no load conditions ( 0.88 S.D. 0.48 ) had comparable performances $F(1,20)$ $=2.04$, partial $\eta^{2}=0.093, p=0.17$, and comparable slopes (0.34 S.D. 0.42 for spatial load and 0.22 S.D. 0.20 for the no load) $\mathrm{T}(21)=-1.2, \mathrm{p}=.26$ (Figure 1).

\section{Discussion}

It has long been established that our ability to enumerate visual stimuli varies as a function of the number of items presented $[11,13,14,15,25,26]$. However, the role of sub-systems of working memory in distinct enumeration processes remains unknown. Therefore, the main goal of the present study was to examine the respective roles of working memory sub-systems in the enumeration processes of subitizing and serial counting.

A common conceptualization of working memory is based on Baddeley's model $[16,17]$ that proposed a partitioning of working memory into three components, namely the central executive, phonological loop, and visual-spatial sketchpad. Additional models suggested that visuo-spatial sketchpad is not a unitary ability and that dissociations can be found between visual and spatial working memory tasks $[21,22]$. In accordance with this hypothesis, the respective roles of phonological and spatial working memory were examined in an enumeration task.
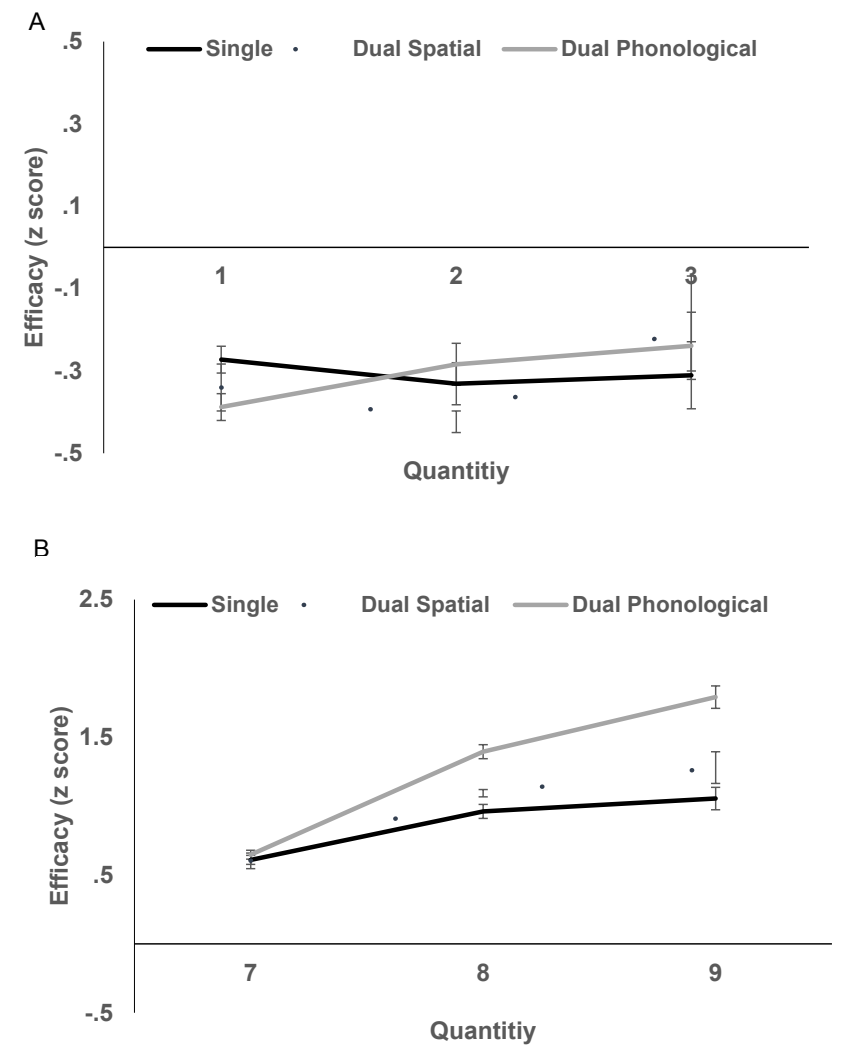

Figure 1: Counting range, participants had better performance in the no load condition as compared with the phonological load condition.
To explore the functions of working memory in enumeration, a dual- task paradigm was employed in which phonological load and spatial load were added to an enumeration task. Importantly, the difficulty of the working memory portion of the dual task was matched to the participants own ability in the phonological and spatial working memory tasks.

The main finding of the current study was that phonological load, as opposed to spatial load, negatively impacted performance in the enumeration task in the serial counting range but not the subitizing range, thus, demonstrating the involvement of different cognitive processes underlying subitizing and serial counting. Specifically, phonological working memory seems to play a significant role in serial counting but not subitizing. Additionally, and contrary to our predictions, subitizing was not affected by spatial working memory load.

\section{Subitizing and counting are based on different cognitive mechanisms}

The present findings, along with previous findings, clearly demonstrate dissociation between subitizing and serial counting $[11,13-15,25,26]$. While serial counting is supported by phonological working memory, subitizing is not.

Serial counting is a more complex process than subitizing and requires individualization and localization of objects, switching spatial attention from one object to the other, vocally summing the number of objects and inhibition of the already summed objects. Phonological working memory will be needed for all of the stages on serial counting. However, enumeration in the subitizing range requires fewer steps. The number is identified and individualized simultaneously: the only requirement for phonological memory in subitizing is access to the single number name. Hence, it comes as no surprise that serial counting but not subitizing suffers from phonological load.

Despite the impact of phonological load on serial counting, there was still a clear distinction between subitizing and serial counting in the participant's performances across load conditions. Discontinuities in the curve were apparent in both dual task conditions: the slope in the subitizing range was flat and increased linearly in the serial counting range. This suggests that the load in the dual task condition did not tap out the participants' resources enough to prevent subitizing and serial counting. This suggests that the emergence of subitizing and counting is unaffected by manipulations designed to limit spatial and phonological working memory resources.

\section{Subitizing is not affected by spatial working memory load}

Contrary to the idea that small numerosities are processed by an object individuation mechanism supported by spatial working memory, we observed that subitizing did not suffer from spatial load. Similarly, [11] examined subitizing capacities using the dual- task paradigm. Their study combined a naming task with a visual working memory task; first, participants were presented with a memory set of circles in varied colors and locations. Then participants performed the enumeration task. After the enumeration task, participants were presented with an additional set of circles and had to report whether it was the same or different than the one displayed at the beginning of the trial. All the circles were presented simultaneously and varied only in colors, manipulating visual working memory. The result indicated that under visual working memory load subitizing suffered.

However, in the present study, we used the frequently used test 
of spatial working memory the 'Corsi block tapping task' for the manipulation of cognitive load [27], which requires serial coding of spatial locations. Recent models suggest that visuo-spatial working memory is not a unitary ability and that it can be further divided to visual (remember details about the object such as color) and spatial (remember the location of the object) working memory tasks [21,22].

In the last 15 years the study of visual working memory developed independently from spatial working memory [28]. Visual information but not spatial information is typically acquired during short fixations and then saccadic eye movements that temporarily suppress processing and then shift the retinal image. Memory is needed to bridge the temporal gaps and spatial shifts created by eye movements [28]. In line with this hypothesis, it was found that spatial working memory load but not visual working memory load decreased the effectiveness of visual search [21].

Furthermore, neuroanatomical evidence noticeably reveals dissociation between visual and spatial working memory abilities. Specifically, visual coding relies on the dorsal stream located in occipitaltemporal pathway which encodes visual information regarding the object, while spatial coding is dependent on the inferior parietal cortex [18]. In line with this distinction, lateral occipital and calcirine damage selectively impaired subitizing but not serial counting [29]. In contrast, impairments in serial counting were associated with damage to the left intraparietal sulcus (but not in subitizing) [29].

The findings of the current study, along with the findings of Piazza et al. [11], provide strong evidence for the mechanisms underlying enumeration processes and perhaps the neuroanatomical structures that support these processes. Subitizing is supported by a domain general visual object individuation mechanism and not a spatial working memory mechanism. It is proposed that subitizing is supported by occipital-temporal areas but not the inferior parietal cortex.

\section{Conclusions}

It has long been established that our ability to enumerate visually presented stimuli varies as a function of the number of items presented [11,13-15,25,26]. However, the role of sub-systems of working memory in distinct enumeration processes has remained unknown.

In the present study, the dual task paradigm was employed in order to examine the roles of phonological and spatial working memory in the enumeration processes of subitizing and serial counting [11,13-15,25,26]. In line with the majority of the findings regarding enumeration processes, the results indicate that enumeration processes of small and large quantities are based on differentiated cognitive processes.

Moreover, I discovered distinct processes of subitizing and serial counting even in the dual task condition, thus suggesting that both phonological and spatial working memory are not essential components of enumeration processes. Serial counting suffered from phonological but not spatial load, while subitizing was not influenced from working memory load manipulation. Accordingly, phonological working memory plays a significant role in serial counting but not in subitizing.

\section{References}

1. Akin O, Chase W (1978) Quantification of three-dimensional structures. Journal of experimental psychology. Human perception and performance 4: 397-410.

2. Mandler G, Shebo BJ (1982) Subitizing: an analysis of its component processes. J Exp Psychol Gen 111: 1-22.

3. Simon TJ, Peterson S, Patel G, Sathian K (1998) Do the magnocellular and parvocellular visual pathways contribute differentially to subitizing and counting? Percept Psychophys 60: 451-464.

4. Trick LM, Pylyshyn ZW (1993) What enumeration studies can show us about spatial attention: evidence for limited capacity preattentive processing. Journal of experimental psychology. Human perception and performance, 19: 331-351.

5. Kaufman EL, Lord MW (1949) The discrimination of visual number. Am J Psychol 62: 498-525.

6. Dehaene S, Cohen L (1994) Dissociable mechanisms of subitizing and counting: neuropsychological evidence from simultanagnosic patients. Journal of experimental psychology. Human perception and performance 20: 958-975.

7. Gallistel CR, Gelman R (1992) Preverbal and verbal counting and computation Cognition 44: 43-74.

8. Feigenson L, Carey S (2005) On the limits of infants' quantification of small object arrays. Cognition 97: 295-313.

9. Feigenson L, Dehaene S, Spelke E (2004) Core systems of number. Trends Cogn Sci 8: 307-314

10. Revkin SK, Piazza M, Izard V, Cohen L, Dehaene S (2008) Does subitizing reflect numerical estimation? Psychol Sci 19: 607-614.

11. Piazza M, Fumarola A, Chinello A, Melcher D (2011) Subitizing reflects visuospatial object individuation capacity. Cognition 121: 147-153.

12. Pylyshyn Z (1989) The role of location indexes in spatial perception: a sketch of the FINST spatial-index model. Cognition 32: 65-97.

13. Pylyshyn ZW, Storm RW (1988) Tracking multiple independent targets: evidence for a parallel tracking mechanism. Spatial vision 3: 179-197.

14. Railo H, Koivisto M, Revonsuo A, Hannula MM (2008) The role of attention in subitizing. Cognition 107: 82-104.

15. Tuholski SW, Engle RW, Baylis GC (2001) Individual differences in working memory capacity and enumeration. Mem Cognit 29: 484-492.

16. Baddeley A (2001) Is working memory still working? Am Psychol 56: 851-864.

17. Baddeley A, Hitch GJ (1974) Working Memory, Elsevier pp: 47-89.

18. Baddeley A (2003) Working memory: looking back and looking forward. Nat Rev Neurosci 4: 829-839.

19. Baddeley A (2010) Working memory. Curr Biol 20: R136-140.

20. Baddeley A (2012) Working memory: theories, models, and controversies. Annu Rev Psychol 63: 1-29.

21. Klauer KC, Zhao Z (2004) Double dissociations in visual and spatial short-term memory. J Exp Psychol Gen 133: 355-381.

22. Mohr HM, Linden DE (2005) Separation of the systems for color and spatia manipulation in working memory revealed by a dual-task procedure. J Cogn Neurosci 17: 355-366.

23. Baddeley A (1992) Working memory. Science 255: 556-559.

24. Cocchini G, Logie RH, Della Sala S, MacPherson SE, Baddeley AD (2002) Concurrent performance of two memory tasks: evidence for domain-specific working memory systems. Mem Cognit 30: 1086-1095

25. Trick LM (2005) The role of working memory in spatial enumeration: patterns of selective interference in subitizing and counting. Psychon Bull Rev 12: 675-681.

26. Trbovich PL, LeFevre JA (2003) Phonological and visual working memory in mental addition. Mem Cognit 31: 738-745.

27. Berch DB, Krikorian R, Huha EM (1998) The Corsi block-tapping task: methodological and theoretical considerations. Brain Cogn 38: 317-338.

28. Luck SJ, Vogel EK (2013) Visual working memory capacity: from psychophysics and neurobiology to individual differences. Trends Cogn Sci 17: 391-400.

29. Demeyere N, Rotshtein P, Humphreys GW (2012) The Neuroanatomy of Visual Enumeration: Differentiating Necessary Neural Correlates for Subitizing versus Counting in a Neuropsychological Voxel-based Morphometry Study. Journal of Cognitive Neuroscience 24: 948-964. 